Trauma Berufskrankh 2010 - 12[Suppl 2]:183-187 DOI 10.1007/s10039-009-1535-9

Online publiziert: 9. Oktober 2009

๑) Springer Medizin Verlag 2009

\author{
A. Woltmann · H. Eckardt · L. Gaul \\ BG-Unfallklinik Murnau
}

\title{
Management der Beckenverletzungen beim Polytrauma
}

Versorgung von Beckenfrakturen weiterhin hohe Ansprüche an die chirurgische Therapie.

\section{"Damage control surgery" in der Beckenchirurgie}

Der Begriff des „damage control“ (DC) wurde erstmalig durch die US-Navy geprägt. Er beschrieb das Vorgehen in einer Situation, die das unweigerliche Sinken eines Schiffes zur Folge hat, mit dem Ziel, den größtmöglichen Schaden abzuwenden. Übertragen auf die Chirurgie bedeutet dies den Verzicht auf eine frühe definitive Versorgung zugunsten lebenserhaltender und -sichernder Sofortmaßnahmen.

Medizinisch wurde das DC erstmalig durch Rotondo et al. [7] 1993 zur Behandlung des abdominalen Traumas bei
Schwerstverletzten beschrieben. Es folgte die Übertragung des Behandlungskonzepts auf die Gebiete der Neuro-, Wirbelsäulen-, Extremitäten- und Thoraxchirurgie $[2,6,8,9]$. „Damage control“ bei der Behandlung von Beckenverletzungen bedeutet, in der Primärphase der Behandlung eine Blutungsreduktion durch Kompression und eine Lagerungsstabilität durch Fixation zu erreichen. Hierzu steht eine Reihe von Hilfsmitteln bzw. operativen Maßnahmen zur Verfügung:

- Beckenschlinge („pelvic sling“)

- Fixateur externe

- Beckenzwinge

- Bauchtuchtamponade

Die Beckenschlinge kommt mittlerweile flächendeckend bereits in der Akutversorgung im Rettungswesen zum Einsatz. arteriellen Gefäßen des kleinen Beckens. Zum anderen bestehen zusätzlich häufig schwerwiegende Begleitverletzungen des Schädels, Thorax oder Abdomens.

Im Rahmen der interdisziplinären Behandlung des polytraumatisierten $\mathrm{Pa}$ tienten ist die Schwere der Begleitverletzungen [z. B. Schädel-Hirn-Trauma (SHT)] oft für das therapeutische Vorgehen entscheidend. Die Prognose und Mortalität werden in der Regel durch die Begleitverletzungen bestimmt $[1,4]$. Aus diesen Gründen ist in der Primärphase der Therapie die Kreislaufstabilisierung vorrangiges Prinzip. Die frühe, zeitaufwändige, definitive Versorgung der Beckenfraktur tritt meist zugunsten des „damage control“ in den Hintergrund. Aufgrund der Komplexität der Beckenund Frakturanatomie stellt die operative

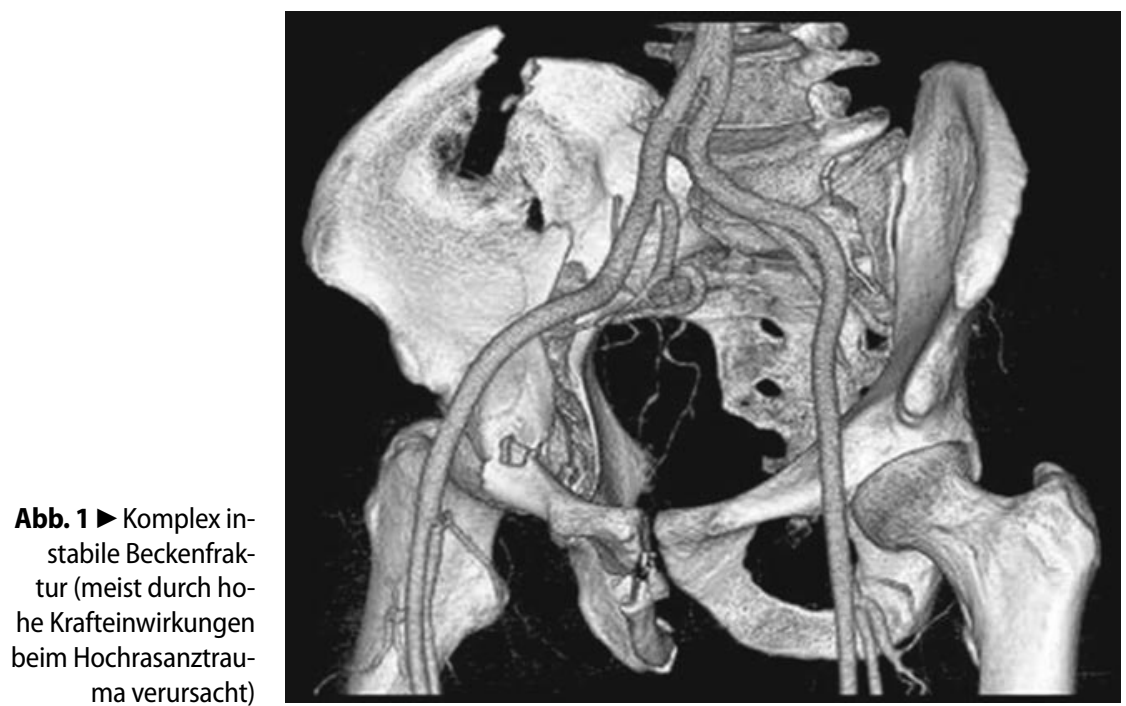




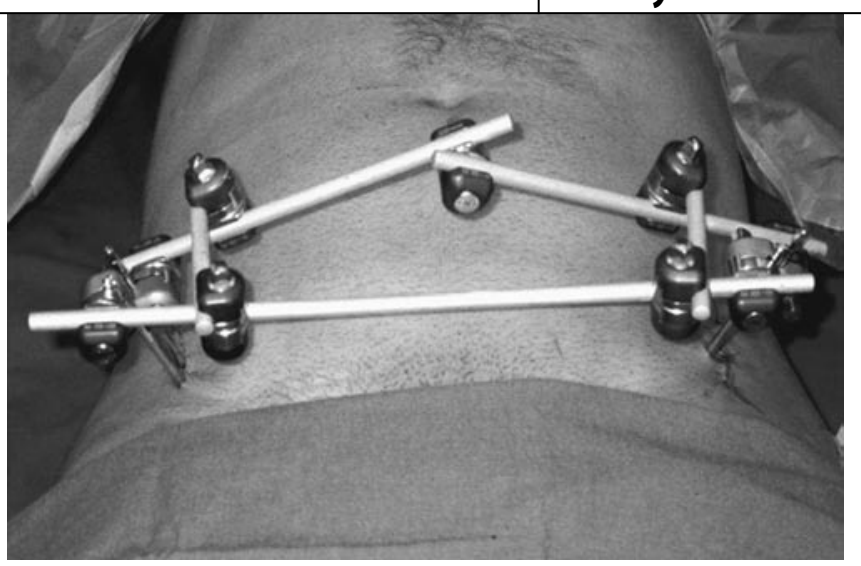

Abb. $2 \varangle$ Beckenfixateur als schnelles und einfaches Stabilisierungsverfahren zur Gewährleistung von Lagerungsstabilität und Kompression

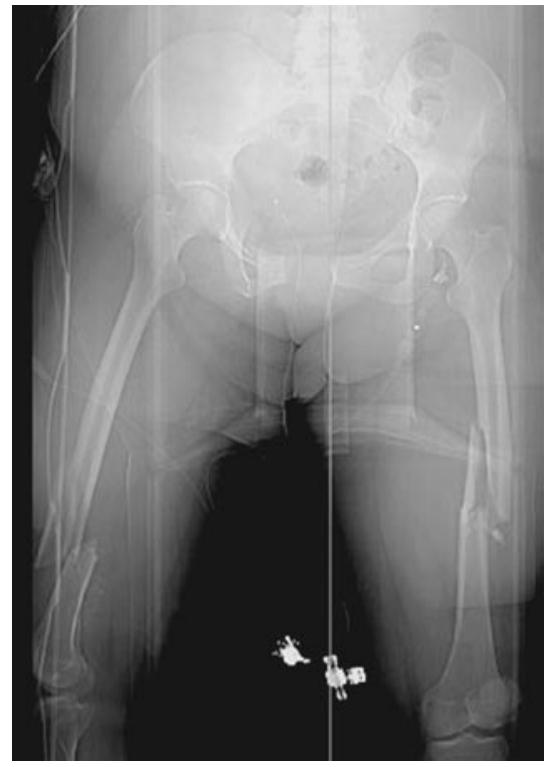

Abb. $3 \Delta$ Multiple Extremitätenverletzungen mit Körperstammverletzung als typische, ein Vorgehen im Sinne des "damage control" indizierende Begleitverletzung

Ein operatives Vorgehen ist für den Einsatz des Fixateur externe, der Beckenzwinge sowie zur offenen Blutungskontrolle notwendig. Ziel der externen Stabilisierung ist es, eine Autotamponade besonders venöser Blutungen aus dem pelvinen und sakralen Plexus zu erreichen und eine kreislaufstabile Situation zur sekundären definitiven Osteosynthese zu schaffen. In unserer Klinik hat sich diesbezüglich die Anlage des Fixateur externe ( $\bullet$ Abb. 2) als schnelles und technisch einfaches Verfahren als operativer Standard etabliert (etwa 10-15 min Operationszeit). Neben der Lagerungsstabilität ist die erleichterte Mobilisation in der Frühphase auf der Intensivstation von Vorteil.
Selten ist bei durch externe Kompression nicht zu stillender Blutung ein offenes Vorgehen mit Bauchtuchtamponade notwendig.

Die Embolisation oder Ligatur arterieller Gefäße sind nur in Ausnahmefällen erforderlich.

\section{Entscheidungskriterien zum "damage control"}

Die Indikationen zu einem Vorgehen im Sinne des „damage control“ bei Beckenverletzungen beim Polytraumatisierten sind:

- Schwierige Kreislaufstabilisierung [ $>5$ Erythrozytenkonzentrate (EK), Thrombozyten $<100.000 / \mu$ l, Temperatur $<34^{\circ} \mathrm{C}$ ]

- Schweres Schädel-Hirn-Trauma [GCS $<8$ (Glasgow coma scale)] mit Blutung/Schwellung

- Thoraxtrauma mit bilateralen Lungenkontusionen [AIS >3 (,acute injury severity")]

- Verletzung großer Extremitätenarterien

- Multiple Extremitätenverletzungen in Verbindung mit Körperstammverletzung und/oder SHT (• Abb. 3)

\section{Definitive osteosynthetische Versorgung}

Sie richtet sich nach der Klassifikation und dem Grad der Instabilität der Beckenfraktur. Das funktionelle Ziel liegt in der Herstellung der Belastbarkeit und der bestmöglichen anatomischen Rekonstruktion der Beckenanatomie. Dies erfolgt unter Rücksichtnahme auf die bei Belastung auftretenden hohen Kräfte im Bereich insbesondere des hinteren Beckenrings.

Vor diesem Hintergrund ist der Zeitpunkt zur definitiven Osteosynthese so zeitnah wie möglich und so spät wie nötig zu wählen. In der Literatur ist eine geringere Mortalität für eine Operation nach dem 3. Tag beschrieben [5]. Nur selten können eine primäre offene Reposition und Stabilisierung der Fraktur notwendig werden, $z$. B. bei:

- Vorliegen einer begleitenden intraabdominalen Harnblasenruptur

- Vorliegen einer verhakten Luxation des Hüftgelenks

- fehlender Möglichkeit einer externen Stabilisierung

- Vorliegen einer zusätzlich zu stabilisierenden Lendenwirbelkörperfraktur

Aufgrund der Frakturinstabilität erfordern annähernd alle Becken-C- und ein Teil der Becken-B-Frakturen eine osteosynthetische Versorgung. Im Bereich des vorderen Beckenrings kommt häufig die Transfixation der Symphysensprengung durch Platte zum Einsatz. Zur Stabilisierung des hinteren Beckenrings und bei möglicher geschlossener Reposition haben sich die perkutane Transfixation des Iliosakralgelenks bzw. die Osteosynthese von lateralen Sakrumlängsfrakturen durch kanülierte Schrauben bewährt. Ist eine offene Reposition notwendig, wird in dieser Lokalisation die Plattenosteosynthese durchgeführt. Zusätzlich kommt bei Sakrumtrümmerfrakturen mit großer Defektzone und/oder begleitenden Wirbelkörperfrakturen die iliolumbale Transfixation zur Anwendung. Der primär angelegte Fixateur externe kann entsprechend der Frakturmorphologie entweder als auxiliärer Fixateur belassen (z. B. C-Verletzung mit vorderem Beckenring) oder im Sinne eines Verfahrenswechsels (z. B. auf Symphysenplatte bei B1-Verletzung) entfernt werden.

\section{Untersuchung anhand eigener Daten}

Zur Analyse der Versorgungsstrategien der Beckenfraktur beim Polytrauma wurden retrospektiv die Daten sämtlicher in den Jahren von 2003-2008 in unserer Klinik behandelter polytraumatisierter Pati- 
enten mit Becken- und/oder Azetabulumfraktur erhoben. Erfasste Parameter waren u. a.

- Unfallmechanismus

- Alter

- Klassifikation [AO-Klassifikation (AO: Arbeitsgemeinschaft für Osteosynthesefragen)]

- ISS („injury severity score“)

- Begleitverletzung (AIS nach Organsystem: Schädel, Thorax, Abdomen, Wirbelsäule, Extremitäten)

- Mortalität

- Operationszeitpunkt und -technik

- Intensiv- und Klinikaufenthalt

\section{Verletzung}

Bei 18\% (220/1215) aller polytraumatisierten Patienten waren eine Becken- und/ oder Azetabulumfraktur vorhanden. Die Mortalität betrug 6\%, 114 Patienten (51\%) wurden operiert. Hierbei waren in 142/220 der Fälle isoliert das Becken, in 50/220 das Azetabulum sowie in 28/220 der Fälle Becken und Azetabulum betroffen.

Der Anteil der stabilen Beckenfrakturen (AO-Typ A) betrug 18\%, der B-Verletzungen $35 \%$ sowie der C-Verletzung $47 \%$. Beim Azetabulum lagen in 56\% A-, in $39 \% \mathrm{~B}$ - sowie in $5 \% \mathrm{C}$-Verletzungen vor.

\section{Mortalität und ISS}

Es bestand kein Zusammenhang zwischen der Instabilität bzw. Klassifikation der Beckenverletzung und der Mortalität, jedoch zwischen der Gesamtverletzungsschwere (ISS) und der Mortalität (• Tab. 1, 2). Diese Ergebnisse reflektieren die aktuelle Studienlage [3, 4]. Die Mortalität wurde überwiegend durch das Schädel-HirnTrauma bestimmt.

\section{Begleitverletzungen}

Die Analyse der Begleitverletzungen zeigte, dass am häufigsten Extremitäten(100\%) und Thoraxverletzungen (68\%) vorlagen (- Tab. 3). Ein Unterschied in der Verteilung der Begleitverletzungen zwischen Azetabulum- und Beckenfrakturen bestand nicht. Abdominaltraumen schließen die Verletzungen des Urogenitaltrakts mit ein.

Trauma Berufskrankh 2010 · 12[Suppl 2]:183-187 DOI 10.1007/s10039-009-1535-9

c) Springer Medizin Verlag 2009

\section{A. Woltmann · H. Eckardt · L. Gaul \\ Management der Beckenverletzungen beim Polytrauma}

\section{Zusammenfassung}

Beckenfrakturen beim polytraumatisierten Patienten stellen schwere Verletzungen mit einem hohen Anspruch an die chirurgische Behandlung dar. Aufgrund der hohen Koinzidenz schwerer Begleitverletzungen sind in den meisten Fällen eine primäre Stabilisierung der Fraktur und Kompression der Blutung durch äußere Fixation (,damage control") bis zur sekundären definitiven Osteosynthese notwendig. In der Erhebung eigener Daten aller Polytraumatisierten in den Jahren 2003-2008 betrug der Anteil an Becken-/Azetabulumfrakturen 18\% (220/1215), $51 \%$ der Patienten wurden operiert. Eine Korrelation zwischen Instabilität bzw. AIS („ab- breviated injury scale") der Beckenverletzung und der Mortalität bestand nicht, jedoch zwischen der Gesamtverletzungsschwere und der Mortalität. In 41/114 operativ versorgten Beckenfrakturen wurde ein Fixateur externe als primäre Maßnahme im Sinne des „damage control" angelegt. Die definitive Osteosynthese erfolgte zeitversetzt nach Kreislaufstabilisierung (im Mittel 3-4 Tage nach dem Unfall). In 10\% war die definitive Osteosynthese bereits am Unfalltag erforderlich.

\section{Schlüsselwörter}

Beckenfraktur · Polytrauma - Begleitverletzungen · "damage control" · Osteosynthese

\section{Management of pelvic fractures in polytrauma}

\section{Abstract}

Pelvic fractures are often caused by high-energy blunt trauma in young patients. The prognosis is strongly dependent on the associated injuries, and life-saving procedures have priority in the initial treatment. Immediate external stabilization and compression of the pelvis is required ("damage control") to stop bleeding and allow for safe patient positioning. We analyzed the data of 1,215 polytrauma patients treated at the Trauma Center Murnau (Germany) from 2003 to 2008. Among all patients, $220(18 \%)$ sustained a fracture of the pelvis and acetabulum, and
$51 \%$ required operative stabilization. Mortality correlated with the Injury Severity Score but not with fracture instability (AO classification). In 41 out of 114 patients who were treated operatively, initial fracture stabilization was performed using the external fixator as the primary tool. Definitive osteosynthesis was performed an average of 3-4 days following trauma, and on the first day in $10 \%$.

\section{Keywords}

Polytrauma · Pelvic fracture - Associated injuries - Damage control - Osteosynthesis 


\section{Polytrauma - Aktuell}
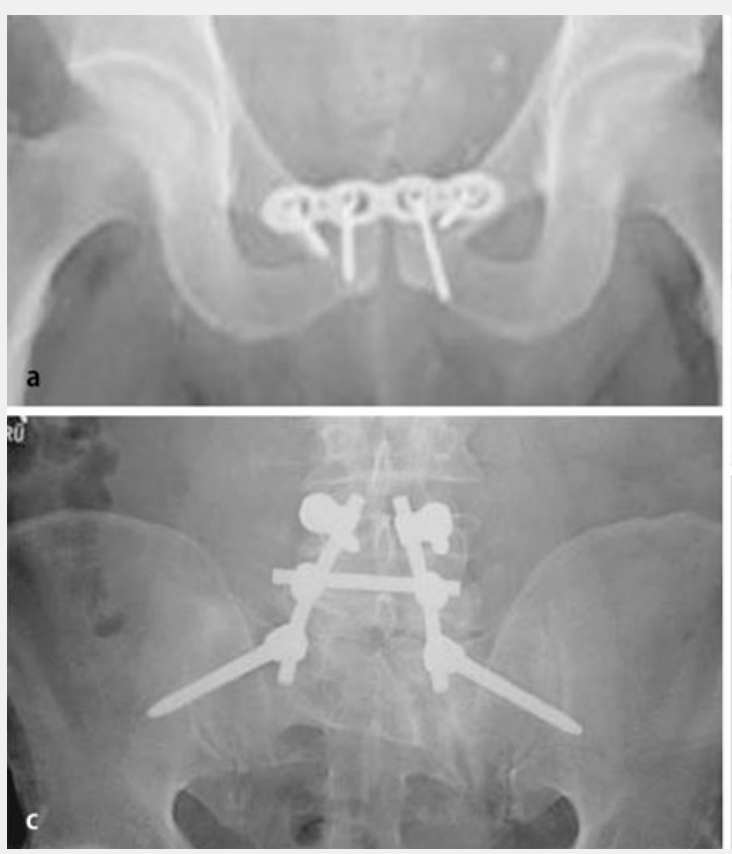

b

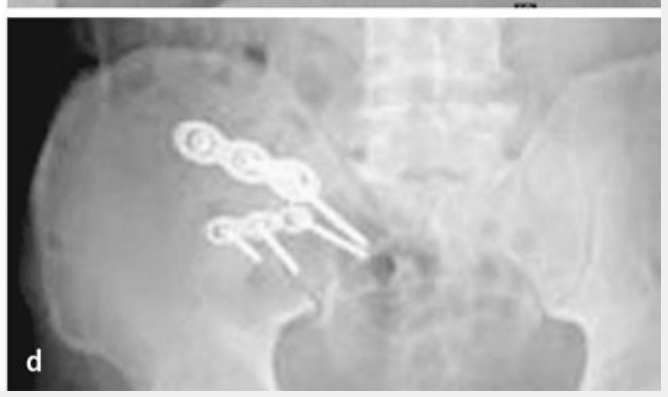

Abb. $4<$ Definitive Osteosynthese des vorderen und hinteren Beckenrings, a Symphysenplatte, b ISG-Schraube, c iliolumbale Transfixation, $\mathbf{d}$ dorsale Plattenosteosynthese
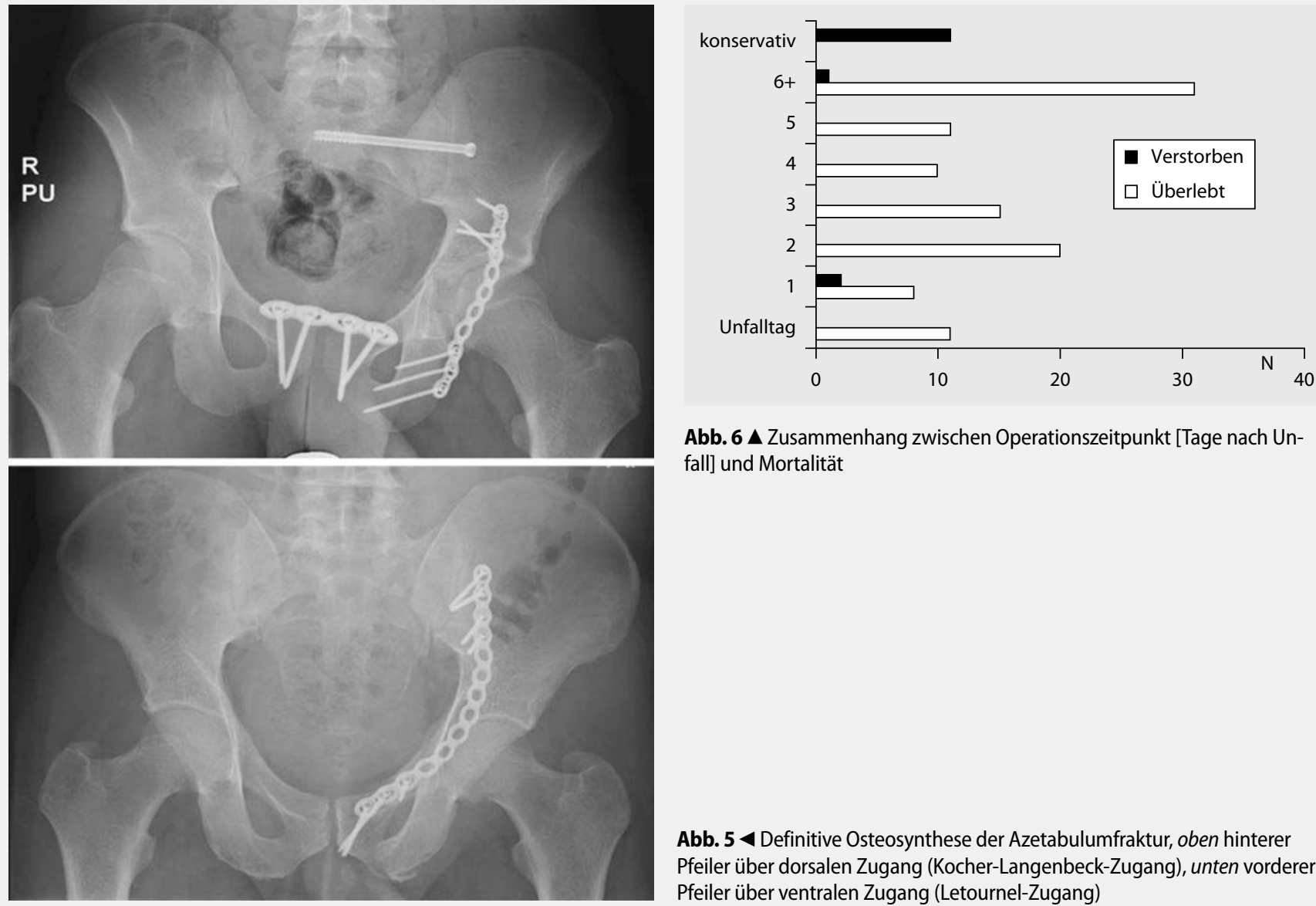

Abb. $6 \Delta$ Zusammenhang zwischen Operationszeitpunkt [Tage nach Unfall] und Mortalität

Abb. $5<$ Definitive Osteosynthese der Azetabulumfraktur, oben hinterer Pfeiler über dorsalen Zugang (Kocher-Langenbeck-Zugang), unten vorderer Pfeiler über ventralen Zugang (Letournel-Zugang) 
Tab. 1 Schweregradabhängige Mortalität aller Polytraumapatienten mit Becken-/Azetabulumfraktur ${ }^{\mathrm{a}}$

\begin{tabular}{lll} 
AIS & Gesamt & Verstorben \\
2 & 65 & 3 \\
3 & 43 & 3 \\
4 & 83 & 4 \\
5 & 29 & 3 \\
$\begin{array}{l}\text { AIS } \text { ",acute injury severity } \\
\text { aMortalität in Abhängigkeit von der Verletzungs- } \\
\text { schwere der Becken-/Azetabulumfraktur }\end{array}$ \\
\hline
\end{tabular}

Tab. 2 SHT-abhängige Mortalität aller

Polytraumapatienten mit Becken-/Azetabulumfraktura

\begin{tabular}{|c|c|c|}
\hline $\begin{array}{l}\text { Polytrauma } \\
\text { Becken }\end{array}$ & Verstorben & Überlebt \\
\hline ISS (Mittel) & 50 & 31 \\
\hline (SHT) [\%] & 58 & 35 \\
\hline AIS SHT (Mittel) & 4,4 & 3,1 \\
\hline \multicolumn{3}{|c|}{$\begin{array}{l}\text { AIS „acute injury severity“, ISS „, injury severity } \\
\text { score”, SHT Schädel-Hirn-Trauma }{ }^{a} \text { Mortalität in Ab- } \\
\text { hängigkeit von ISS, Schwere und Häufigkeit eines } \\
\text { Schädel-Hirn-Traumas }\end{array}$} \\
\hline
\end{tabular}

8. Scalea TM, Boswell SY, Scott JD et al (2000) External fixation as a bridge to intramedullary nailing for patients with multiple injuries and with femur fractures: damage control orthopaedics. J Trauma 48:613-623

9. Wall Jr MJ, Soltero E (1997) Damage control for thoracic injuries. Surg Clin North Am 77:863-878

\section{Tab. 3 Häufigkeit der Begleitverletzungen}

\begin{tabular}{llll}
\hline Polytrauma & Azetabulum [\%] & Becken [\%] & Azetabulum und Becken [\%] \\
Schädel & 34 & 37 & 36 \\
\hline Thorax & 74 & 65 & 68 \\
\hline Abdomen & 29 & 30 & 29 \\
\hline Wirbelsäule & 45 & 42 & 43 \\
\hline Extremitäten & 100 & 100 & 100 \\
Urogenitale Verletzungen werden unter Abdominalverletzungen subsummiert
\end{tabular}

\section{Operative Stabilisierung}

Insgesamt wurden 114 Patienten mit Beckenfraktur und Azetabulumfraktur operiert. Bei 41 Patienten wurde primär ein Fixateur externe angelegt, 8 wurden damit ausbehandelt. Bei 14 Patienten wurde der Fixateur als auxiliäre Stabilisierung des vorderen Beckenrings belassen.

Der Verfahrenswechsel auf interne Osteosynthese erfolgte in der Regel nach 34 Tagen. Zur definitiven Osteosynthese der Beckenfrakturen wurde in je 36 Fällen die Symphysenplatte und die ISG-Schraube (ISG: Iliosakralgelenk), in 15 Fällen die iliolumbale Transfixation sowie in 10 Fällen die dorsale Plattenosteosynthese verwendet (- Abb. 4). Bei Azetabulumfrakturen erfolgten 20 -mal die dorsale und 17mal die ventrale Osteosynthese mit Platte und Zugschrauben/Cerclage (• Abb. 5).

Eine Korrelation zwischen der Mortalität und dem Operationszeitpunkt ergab sich nicht, die Mehrzahl der verstorbenen Patienten wurde aufgrund ihrer schweren Begleitverletzungen keiner definitiven Osteosynthese unterzogen (• Abb. 6). In 14 Fällen (10\%) wurde diese bereits am Unfalltag durchgeführt.

\section{Korrespondenzadresse}

Prof. Dr. A. Woltmann

BG-Unfallklinik Murnau,

Prof.-Küntscher-Straße 8, 82418 Murnau

woltmann@bgu-murnau.de

Interessenkonflikt. Der korrespondierende Autor gibt an, dass kein Interessenkonflikt besteht.

\section{Literatur}

1. Hauschild O, Strohm PC, Culemann U et al (2008) Mortality in patients with pelvic fractures: results from the German pelvic injury register. J Trauma 64:449-455

2. Kossmann T, Trease F, Freedman I, Malham G (2004) Damage control surgery for spine trauma. Injury 35:661-670

3. Lunsjo K, Tadros A, Hauggaard A et al (2007) Associated injuries and not fracture instability predict mortality in pelvic fractures: a prospective study of 100 patients. JTrauma 62:687-691

4. Poole GV, Ward EF (1994) Causes of mortality in patients with pelvic fractures. Orthopedics 17:691696

5. Probst C, Probst T, Gaensslen A et al (2007) Timing and duration of the initial pelvic stabilization after multiple trauma in patients from the German trauma registry: is there an influence on outcome? The Polytrauma Study Group of the German Trauma Society. JTrauma 62:370-377

6. Rosenfeld JV (2004) Damage control neurosurgery. Injury 35:655-660

7. Rotondo M, Schwab CW, McGonigal MD et al (1993) Damage control - an approach for improved survival in exsanguinating penetrating abdominal injury. J Trauma 35:375-382 